\title{
A NEW SOLUTION TO THE WORD PROBLEM IN THE FUNDAMENTAL GROUPS OF ALTERNATING KNOTS AND LINKS
}

\author{
BY
}

MARK J. DUGOPOLSKI

\begin{abstract}
A new solution to the word problem for alternating knots and links is
\end{abstract} given. The solution is based on Waldhausen's algorithm, but is greatly simplified.

I. Introduction. The conjugacy problem, and hence the word problem for alternating knots, was solved by Appel and Schupp [1], and Weinbaum [3], using algebraic methods. Waldhausen [2] gave an algorithm for determining if a loop in a certain type of 3-manifold bounds a singular disk, thus solving the word problem in the fundamental groups of those manifolds. Although Waldhausen's algorithm applies to knots and links, it is extremely difficult to apply. One must first find, using an algorithm of Haken, a hierarchy of surfaces for the 3-manifold which splits it into balls. To do this, one must find nonnegative, integral solutions to a rather large system of equations.

In this paper we show that in the complementary spaces of alternating knots and links, we do not need to use Haken's algorithm to obtain the surfaces. The surfaces are readily available from a projection of the knot or link. By obtaining the surfaces geometrically, we get a better understanding of how the word problem is solved for alternating knots and links.

II. A preliminary result from graph theory. The most difficult part of the proof can be reduced to a question about planar graphs. For clarity we have separated this from the rest of the proof and will take care of it before proceeding to the algorithm.

A loop in a graph is a finite sequence $a_{0}, a_{1}, \ldots, a_{n-1}$ of oriented edges, such that the terminal vertex of $a_{i}$ is the initial vertex of $a_{i+1}(\bmod n)$, and for each $i, a_{i}$ and $a_{i+1}(\bmod n)$ are different edges. Suppose we have a finite sequence of symbols and we place them in order around the boundary of a disk. If there is a way to pair the symbols using nonintersecting arcs contained in the disk, such that each arc connects a pair of identical symbols, then the sequence is called a matching sequence.

For a loop in a graph we obtain a finite sequence of symbols as follows. Let the open regions of the complement of the graph be labeled with distinct symbols. We travel around the loop once, in the direction prescribed by the orientation of the edges. We obtain a sequence of symbols, one for each edge of the loop. The first

Received by the editors July 15, 1980 and, in revised form, April 3, 1981.

1980 Mathematics Subject Classification. Primary 57M05; Secondary 57M25, 20F10.

Key words and phrases. Alternating knots, word problem, incompressible surface. 
symbol is the symbol of the region on our left when traversing the first edge, the second symbol is the symbol of the region on our right when traversing the second edge, etc. The symbols in the sequence correspond to the symbols of the regions on alternating sides of the loop. We will call a sequence obtained in this way an alternating sequence corresponding to a loop. A loop will be called a matching loop if the loop has an alternating sequence which is a matching sequence. Diagram 1 shows how a matching sequence might be matched.

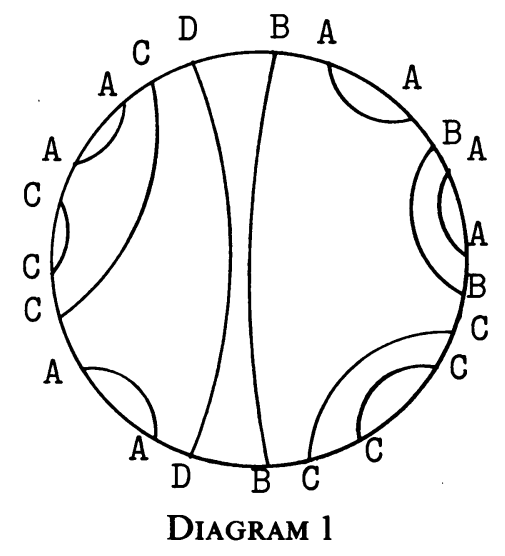

THEOREM 2.1. In a connected planar graph where each edge has two distinct vertices and each vertex has order at least two, there are no matching loops.

Proof of Theorem 2.1. Suppose we have a matching loop. Note that among the arcs used in the matching of a matching sequence, there must be an innermost arc. Thus a matching sequence must have at least one letter occurring twice consecutively. Since consecutive edges of the loop must be different, this means that by traversing these two edges, we find a region $A$ first on one side and then on the other. These two edges have only one vertex in common and its removal would disconnect the graph. Encircle part of the graph with an arc starting at the common vertex and lying in region $A$, as shown in Diagram 2 . We call the region enclosed by this arc a zone.

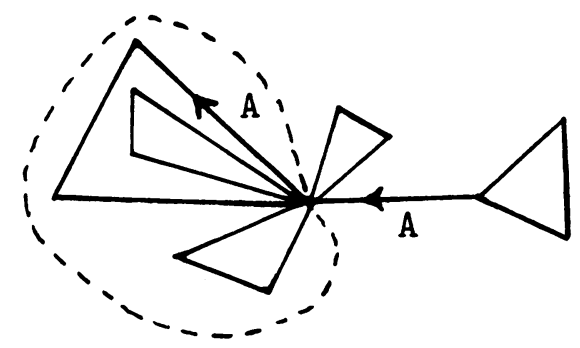

Diagram 2

Each pair of consecutive identical letters occurring in the sequence means the loop is entering a new zone. The only way the loop can leave that zone is to exit at the same vertex where it entered. If the loop enters a zone and then another pair of consecutive identical letters occurs before it exits, the loop is entering a new zone, entirely within the previous zone. 
There must be a zone which is innermost in the sense that no double letters occur in the sequence before the loop exits that zone. Each zone is either innermost or contains an innermost zone. Since no pairs of consecutive identical letters occur within an innermost zone, there can be no matching of letters among those occurring within an innermost zone. Thus when a matching loop enters an innermost zone there must be at least one letter in the sequence that can only be matched by returning to that zone. Now among all such innermost zones choose one where the number of edges of the loop before returning to that zone for the matching is minimal.

Now since the sequence is a matching sequence, there must be a pair of consecutive identical letters among those letters before the return. Choose the first such pair and then the first innermost zone within that zone. This innermost zone must also have a letter that can only be matched by returning to that zone. By the minimality condition we imposed on the first zone, this return must occur after the first return. The arcs used in these matchings must cross. Hence, there can be no matching sequences.

III. The algorithm. All work will be done in the piecewise linear category. Unless stated otherwise, a surface $F$ in a 3-manifold $M$ will be connected and have $F \cap \partial M=\partial F$. The word "disk" will always mean a nonsingular disk. A line over a symbol indicates closure and a small circle is used to indicate interior.

Let $M_{1}$ be a 3-manifold and $F_{1}$ a compact, connected surface in $M_{1}$. We obtain a manifold $M_{2}=\overline{M_{1}-N F_{1}}$ where $N F_{1}$ is a small regular neighborhood of $F_{1}$. We say that $M_{2}$ is $M_{1}$ split at $F_{1}$. There is a natural projection $p_{1}: M_{2} \rightarrow M_{1}$. A finite sequence of such surfaces $F_{1}, \ldots, F_{n}$, where $F_{i} \subset M_{i}$ and each $M_{i}$ is obtained from $M_{i-1}$ by splitting $M_{i-1}$ at $F_{i-1}$, is called a hierarchy of surfaces for the manifold $M_{1}$. We define also the map $P_{i}=p_{1} \circ p_{2} \circ \cdots \circ p_{i}$. At times it is convenient to view all of the surfaces of the hierarchy as lying in $M_{1}$. In this situation, we will often omit the projection maps. For instance, we may write $F_{1} \cap F_{i}=\varnothing$ when we mean $F_{1} \cap P_{i}\left(F_{i}\right)=\varnothing$. Whenever we introduce a new object, we assume it is in general position with respect to all structures already present.

A surface $F$ in a 3-manifold $M$ is called incompressible in $M$ provided that (i) $F$ is not a 2 -sphere, and (ii) if $D$ is a disk in $M$ with $D \cap F=\partial D$, then $\partial D=\partial \tilde{D}$ for some disk $\tilde{D}$ in $F$.

A loop in a 3-manifold $M$ is the image of a map from the 1-sphere $S^{1}$ to $M$. Suppose $l$ is a loop in $M, F$ is a surface in $M$, and $l^{*}$ is an arc of $l$ such that $l^{*} \cap F=\partial l^{*}$. If we know that there is a deformation of $l^{*}$ to $F$ that keeps the endpoints of $l^{*}$ fixed, then we can deform $l$ so that $l$ intersects $F$ in two fewer points. This is called a type 1 reduction of $l$ with respect to $F$. In general, we will use the same letter to denote an object after deformation as before.

Suppose $l^{*}$ is an arc in $M$ such that $l^{*} \cap \partial M=\partial l^{*}, F$ is a surface in $M$ such that $\partial l^{*} \cap F=\varnothing$, and $l_{1}^{*}$ is a subarc of $l^{*}$ such that $l_{1}^{*} \cap F$ consists of one point of $\partial l_{1}^{*}$ and $l_{1}^{*} \cap \partial l^{*}$ is the other point of $\partial l_{1}^{*}$. If we know that $l_{1}^{*}$ deforms to an arc lying in $F \cup \partial M$ intersecting $\partial F$ once, then $l^{*}$ deforms to an arc lying partly in $\partial M$, where the part not in $\partial M$ intersects $F$ one less time than $l$ does. This is called a type 2 deformation of $l^{*}$. See Diagram 3. 


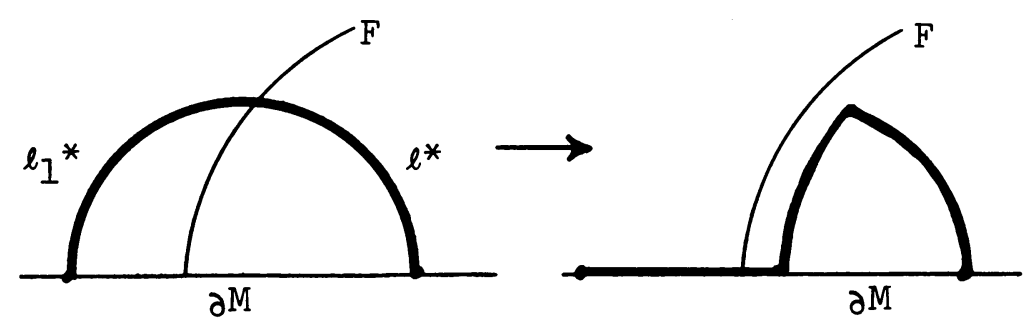

Diagram 3

Suppose $F_{1}, \ldots, F_{n}$ is a hierarchy for $M_{1}$. It will be called a type I hierarchy if the following hold:

1. $F_{1}$ is an incompressible surface which may be orientable or nonorientable, and is not necessarily connected.

2. $F_{2}, \ldots, F_{n}$ are disks such that, for $i \geqslant 2, F_{i} \cap \cup_{2 \leqslant j<i} F_{j}=\varnothing$.

3. If $i \geqslant 2, D$ is a disk, and $f$ is a map from $D$ to $M_{2}$ such that $f^{-1}\left(F_{i}\right)=\beta$, an arc in $\partial D, f^{-1}\left(\cup_{j<i} F_{j}\right)=\varnothing$, and $f^{-1}\left(\partial N F_{1}\right)=\partial D-\beta^{\circ}$, then there is a map $\tilde{f}$ : $D \rightarrow M_{2}$ such that $\left.\tilde{f}\right|_{\beta}=\left.f\right|_{\beta}, \tilde{f}(D) \subset F_{i}$, and $\tilde{f}^{-1}\left(\partial N F_{1}\right)=\partial D-\beta^{\circ}$.

4. If $D$ is a disk and $f$ is a map from $D$ to $M_{1}$ such that $f^{-1}\left(F_{1}\right)=\partial D$, then there exists $f^{\prime}: D \rightarrow M_{1}$ such that $\left.f^{\prime}\right|_{\partial D}=\left.f\right|_{\partial D}$ and $f^{\prime}(D) \subset F_{1}$.

5. $M_{n+1}$ consists only of balls or balls with holes. (A ball with a hole is a ball where a ball disjoint from the boundary of the original ball has been removed.)

THEOREM 3.1. If $M_{1}$ has a type I hierarchy, then there is an algorithm by which we can determine if an arbitrary loop $l$ in $M_{1}$ is contractible or not.

The algorithm is essentially Waldhausen's [2], except that for our hierarchy it is simpler. It differs from Waldhausen's algorithm in allowing a nonorientable surface.

Before we prove Theorem 3.1, we need some preliminaries. We can assume $l$ is nonsingular and in general position with respect to $\bigcup_{1}^{n} F_{i}$ and $\partial N F_{1}$. We also assume that $N F_{1}$ is fibered locally and that if $l \cap N F_{1} \neq \varnothing$, then $l \cap N F_{1}$ consists only of fibers. If $l \cap N F_{1} \neq \varnothing$, then $l$ is split into arcs $l_{1}, \cdots, l_{m}$ by the surface $F_{1}$. Each of these arcs has a small segment at each end which is half of a fiber in $N F_{1}$. Let $l_{i}^{\prime}$ be the subarc of $l_{i}$ which has endpoints on $\partial N F_{1}$ and otherwise does not intersect $N F_{1}$. It is then obvious that there is a deformation of $l_{i}^{\prime}$ to $\partial N F_{1}$ that keeps the endpoints fixed if and only if there is a deformation of $l_{i}$ to $F_{1}$ that keeps the endpoints fixed.

We will need the following lemmas.

LEMMA 3.2. There is an algorithm by which we can check if there is a type 1 reduction that can be performed on $l$ with respect to $F_{1}$.

Proof. Suppose $q$ is an arc such that $q \cap F_{1}=\partial q$, and $q^{\prime}$ is the subarc of $q$ with endpoints on $\partial N F_{1}$. Now $q^{\prime}$ is split into arcs by $\cup_{2}^{n} F_{i}$. Since these arcs lie in the balls of $M_{n+1}$, it is only the position of the endpoints of the arcs that determines whether or not a deformation can be performed. We can check each of these arcs just by looking at $\partial M_{n+1}$ to see if we get a type 1 reduction of $l$ with respect to $F_{i}$, for some $i>1$, or if we get a type 2 deformation. Call this process $X$. 


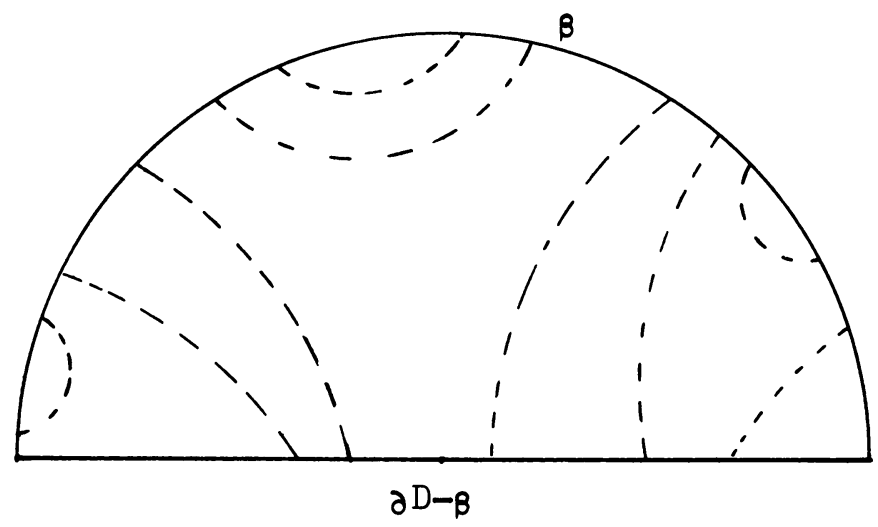

Diagram 4

If $q \cap \cup_{2}^{n} F_{i}=\varnothing$, then we use $X$ to check $q$ for a type 1 reduction of $l$ with respect to $F_{1}$. Since all of the surfaces have Property 4 of the hierarchy, when a type 1 reduction with respect to any surface exists, it can be done in essentially only one way. If $q \cap \cup_{2}^{n} F_{i} \neq \varnothing$, then for every arc of $q^{\prime}$ with endpoints on the same disk, we check for a type 1 reduction of $l$ with respect to that disk. We perform all such reductions, each time reducing the number of points of $q^{\prime} \cap \cup_{2}^{n} F_{i}$ by two.

If now $q \cap \cup_{2}^{n} F_{i}=\varnothing$, we use $X$. If not, let $q_{1}^{\prime}, \ldots, q_{s}^{\prime}$ be the subarcs of $q^{\prime}$. We then use $X$ to check $q_{1}^{\prime}$ for a type 2 deformation. Conditions 3 and 4 of the hierarchy guarantee that a type 2 deformation is essentially unique. If we get a type 2 deformation, we deform $q^{\prime}$ so that it lies partly in $\partial N F_{1}$ and the part which is not in $\partial N F_{1}$, is an arc with endpoints in $\partial N F_{1}$, intersecting $\cup_{2}^{n} F_{i}$ in one point fewer. We then continue to try for the same type of reduction on the new arc, provided the arc still intersects $\cup_{2}^{n} F_{i}$. If it does not, we use $X$ to check for a type 1 reduction on the last arc. If we are successful at each point, we see that $q^{\prime}$ deforms to $\partial N F_{1}$, and hence $q$ deforms to $F_{1}$.

If we fail at any point in this sequence of checking, then $q$ does not deform to $F_{1}$. The reason for this is clear if we consider the map $f: D \rightarrow M_{2}$ such that $f^{-1}\left(q^{\prime}\right)$ is an arc $\beta$ in $\partial D$ and $f(\partial D-\beta) \subset \partial N F_{1}$. We can put $f(D)$ in general position so that $f^{-1}\left(\cup_{1}^{n} F_{i}\right)$ consists of the following kinds of disjoint arcs and simple closed curves:

1. arcs with both endpoints on $\beta$,

2. arcs with both endpoints on $\partial D-\beta$,

3. arcs with one endpoint on $\beta$ and the other on $\partial D-\beta$,

4. simple closed curves.

Using Properties 2-4 of the hierarchy, we can get a $\tilde{f}$ such that $\tilde{f}^{-1}\left(F_{1}\right)$ is empty, $\left.\tilde{f}\right|_{\beta}=\left.f\right|_{\beta}$, the set $\tilde{f}(\partial D-\beta) \subset \partial N F_{1}$, and $\tilde{f}^{-1}\left(\cup_{2}^{n} F_{i}\right)$ consists only of disjoint arcs of types 1 and 3 . It is then obvious that the process will work as described. See Diagram 4, where the dashed lines represent arcs of $\tilde{f}^{-1}\left(\cup_{2}^{n} F_{i}\right)$.

LEMMA 3.3. If $l$ contracts, then the number of times $l$ intersects $F_{1}$ can be reduced to zero by performing all possible type 1 reductions. 
Proof. If $l$ bounds a singular disk, we have a map $f$ from a disk $D$ to $M_{1}$, where $\left.f\right|_{\partial D}$ is $l$. We put $f(D)$ into general position, so that $f^{-1}\left(F_{1}\right)$ consists of disjoint simple closed curves and arcs. Suppose $\alpha$ is an innermost simple closed curve of $f^{-1}\left(F_{1}\right)$. Since $f(\alpha)$ bounds a singular disk in $M_{2}$, Property 4 of $F_{1}$ implies that $f(\alpha)$ bounds a singular disk in $F_{1}$. We move this disk off of $F_{1}$, changing the map $f$ so that $f^{-1}\left(F_{1}\right)$ contains one fewer simple closed curve. In this way we can get a map $f$ : $D \rightarrow M_{1}$, where $\left.f\right|_{\partial D}$ is $l$ and $f^{-1}\left(F_{1}\right)$ consists only of disjoint arcs. This shows us that if we do all possible type 1 reductions, then $l$ will be deformed so that $l \cap F_{1}=\varnothing$.

Proof of Theorem 3.1. If $l \cap F_{1} \neq \varnothing, l$ is split into arcs $l_{1}, \ldots, l_{m}$. Check each arc for a type 1 reduction. If we get one, we reduce $l \cap F_{1}$ by two points and try again. If we cannot get $l \cap F_{1}=\varnothing$ by repeating this, the answer is "no", by Lemma 3.3. If we got $l \cap F_{1}=\varnothing$, then $l$ lies in $M_{2}$ and each component of $M_{2}$ is a handlebody. Since $F_{1}$ is incompressible, we see that $l$ contracts in $M_{2}$ if and only if $l$ contracts in $M_{1}$. We could check algebraically if $l$ represents the trivial element in $\pi_{1}\left(M_{2}\right)$, since $\pi_{1}\left(M_{2}\right)$ is free. However, we can continue to perform all possible type 1 reductions on $l$. It is easy to see that $l$ contracts in $M_{2}$ if and only if $l$ can be freed from intersecting the disks when we apply all possible type 1 reductions.

IV. A new solution to the word problem in the fundamental groups of alternating knots and links. A knot is a piecewise linear homeomorphic image of $S^{1}$ and a link is a disjoint union of finitely many knots. Consider a knot or link in $S^{3}$ and project it onto a plane so that there are only finitely many singularities, all double points. An alternating projection is one where the double points are alternately overcrossings and undercrossings as we travel around the knot. An alternating knot or link is one that has an alternating projection. In this section we will show that for alternating knots and links, the type I hierarchy is readily available from the alternating projection and the algorithm of §III can be applied.

The projection of a knot or link onto a plane separates the plane into open regions. Suppose the regions are colored alternately black and white so that any two regions whose closures share an edge are to be of opposite colors. Let the unbounded region be colored white. In the case where we have a single knot, we can get a spanning surface of the knot from the black regions. It consists of disks joined together by ribbons with a half-twist at every double point. For the projection of the trefoil given in Diagram 5, the black surface is a Möbius band. Of course, different projections may yield different surfaces.

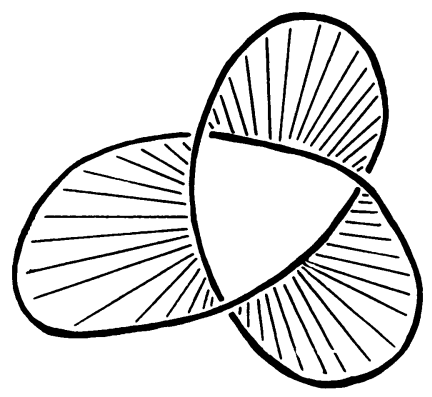

Diagram 5 
THEOREM 4.1. Given an alternating projection for an alternating knot or link $T$, we can obtain a type I hierarchy for the manifold $M_{1}=\overline{S^{3}-N T}$ where NT is a small regular neighborhood of $T$.

Proof. If we let $F_{1}$ be the black surface formed from the coloring of the regions, then $M_{1}-N F_{1}$ is a handlebody. The disks which split $M_{2}$ into a ball come from the white regions of the projection. See Diagram 6.

We first show that $F_{1}$ satisfies Condition 4 of a type I hierarchy. We may assume that the closure of every black region is simply connected, since this could be accomplished by untwisting any section where this fails to hold. $F_{1}$ may consist of several components, but it suffices to prove the theorem for $F_{1}$ connected.

We view $F_{1}$ as a collection of disks connected by strips, each given a half-twist. Since the projection is alternating, all twists are in the same direction. We view $f(\partial D)$ as a path which travels between the disks, crossing the strips to get from disk to disk. We may assume $f(\partial D)$ has been straightened on the strips, so that all singularities lie in the disks. We may also assume that $f(\partial D)$ has been simplified by homotopy so that it does not go from disk $D_{1}$, to a disk $D_{2}$, then immediately back to $D_{1}$ over the same strip. If the path does not cross any strips, then it contracts in $F_{1}$. We will thus show that crossing at least one strip is impossible for a path simplified as we have just described. To prove this, we transform the problem into an equivalent problem in graph theory.

The disks which form $F_{1}$ are replaced by vertices, and the ribbons connecting the disks are replaced by edges connecting the appropriate vertices. This gives us a planar graph in which every edge has two distinct vertices and each vertex is at least of order two. The path $f(\partial D)$ then becomes a loop in the graph as in §II.

Now $f(D)$ is in $M_{1}-F_{1}$ and $M_{1}-F_{1}$ is split into a ball by the disks $F_{2}, \ldots, F_{n}$. Whenever $f(\partial D)$ crosses a strip in $F_{1}, f(\partial D)$ intersects one of the disks $F_{2}, \ldots, F_{n}$. In terms of the graph, $f(\partial D)$ intersects a disk for each edge it crosses, but because the projection is alternating, it alternately intersects the disks on the left or right side of the edge it is crossing.

In $D, f^{-1}\left(\cup_{2}^{n} F_{i}\right)$ consists of arcs whose endpoints are on $\partial D$. The points of $f^{-1}\left(f(\partial D) \cap \cup_{2}^{n} F_{i}\right)$ on the boundary of $D$ are matched with the arcs of $f^{-1}\left(\cup_{2}^{n} F_{i}\right)$.

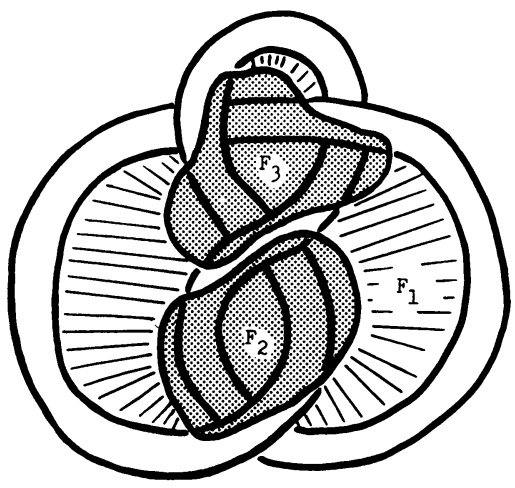

Diagram 6 
The situation we are in is precisely the one described in §II. By Theorem 2.1, $f(\partial D)$ must be contained in one disk of $F_{1}$ and cannot cross any ribbons. Thus $f(\partial D)$ contracts in $F_{1}$ and we have established Property 4. This also gives us Property 1.

For Property 3 of the hierarchy, suppose we have a disk $D$ and a map $f: D \rightarrow M_{2}$ such that $f^{-1}\left(F_{i}\right)$ is an $\operatorname{arc} \beta$ in $\partial D, f^{-1}\left(\cup_{i<j} F_{j}\right)=\varnothing$, and $f^{-1}\left(\partial N F_{1}\right)=\partial D-\beta^{\circ}$. If the endpoints of $f(\beta)$ are in the same component of $F_{i} \cap \partial N F_{1}$, we are done. If they are in different components, then $\partial F_{i}-f(\partial \beta)$ consists of two arcs, say $d_{1}$ and $d_{2}$. Consider $f(\partial D-\beta) \cup d_{1}$. This is a loop in $\partial M_{2}$ which contracts in $M_{2}$. The endpoints of $f(\beta)$ are in different black disks of the projection. Thus $p_{1}(f(\partial D)-\beta)$ is a path in $F_{1}$, running across at least one twist even after it is simplified. An argument similar to that used to establish Property 4 will show that this is impossible. Thus Property 3 is satisfied and the other properties are obvious. Thus the proof of Theorem 4.1 is complete.

\section{REFERENCES}

1. K. Appel and P. Schupp, The conjugacy problem for the group of any tame alternating knot is solvable, Proc. Amer. Math. Soc. 33 (1972), 329-336.

2. F. Waldhausen, The word problem in fundamental groups of sufficiently large irreducible three-manifolds, Ann. of Math. (2) 88 (1968), 272-280.

3. C. Weinbaum, The word and conjugacy problem for the knot group of any tame, prime, alternating knot, Proc. Amer. Math. Soc. 30 (1971), 22-26.

DePartment of Mathematics, SOUTHEASTERn Louisiana University, HaMmond, Louisiana 70401 